\title{
Performance Analysis of SAC-OCDMA Systems Adopting Overlapping PPM Schemes
}

\author{
Hossam M. H. Shalaby, Senior Member, IEEE
}

\begin{abstract}
A fertile technique, for increasing single-user throughput while keeping a constraint on the light pulsewidth, is proposed for spectral-amplitude-coding optical code-division multiple-access (SAC-OCDMA) systems. In this technique, two-level $M$-ary overlapping pulse-position modulation ( $M$-OPPM) scheme is adopted and each user is assigned two orthogonal code sequences to represent these two levels. The code sequences are selected from a minimum cross-correlation code set. The bit error rate (BER) of the proposed system is derived, taking into account the effects of phase-induced intensity noise, shot noise, and thermal noise in addition to the multiple-access interference. The BER performance of this system is compared to other systems adopting $M$-PPM and OOK schemes under same pulsewidth constraints. Our results reveal that, while keeping the BER well below a prescribed threshold, the proposed $M$-OPPM SAC-OCDMA system achieves higher transmission rate as compared to both $M$-PPM and OOK SAC-OCDMA systems under same constraints. Specifically the transmission rate of a single user of the proposed system can be increased by about $34.44 \%$ as compared to traditional systems.
\end{abstract}

Index Terms-Bit-error-rate (BER), modified prime sequence (MPS) codes, on-off keying (OOK), optical code-division multiple access (OCDMA), overlapping pulse-position modulation (OPPM), pulse-position modulation (PPM), spectral-amplitude coding (SAC).

\section{INTRODUCTION}

$\mathbf{O}$ PTICAL CODE-DIVISION MULTIPLE-ACCESS (OCDMA) techniques are becoming competitive candidates for future optical communications networks [1]-[5]. Indeed, recently, many authors have proposed several frameworks for future optical networks adopting OCDMA techniques. For example in [1], Bhuiyan et al. have presented an analytical approach to the performance evaluation of turbulence induced fading on free space OCDMA communications systems. In [2], Tseng and $\mathrm{Wu}$ have adopted super perfect difference (SPD) code in compact spectral-amplitude-coding optical code-division multiple-access (SAC-OCDMA) passive optical networks (PONs). In [3], Chang et al. have studied diversity OCDMA schemes in optical wireless communications systems. In [4],

Manuscript received February 11, 2013; revised April 23, 2013; accepted April 24, 2013. Date of publication April 30, 2013; date of current version May $15,2013$.

The author is with the Department of Electronics and Communications Engineering, Egypt-Japan University of Science and Technology (E-JUST) Alexandria 21934, Egypt, on leave from the Electrical Engineering Department, Alexandria University, Alexandria 21544, Egypt (e-mail: shalaby@ieee.org).

Color versions of one or more of the figures in this paper are available online at http://ieeexplore.ieee.org.

Digital Object Identifier 10.1109/JLT.2013.2260856
Beyranvand and Salehi have proposed a quality of service (QoS) differentiation framework for optical burst switching (OBS) multiservice networks. They have adopted hybrid wavelength division multiplexing and optical code division multiplexing (WDM/OCDM) schemes in order to mitigate the blocking probability of OBS networks. In [5], Choi et al. have proposed and experimentally demonstrated the upstream transmission of WDM/OCDM-PONs using low-cost devices. Out of the different types of OCDMA, spectral-amplitude-coding optical code-division multiple-access (SAC-OCDMA) comes as a low-complexity and cost-effective candidate [6]. This technique has been first proposed by Zaccarin and Kavehrad [7], [8]. Yet, it has been shown to be suffering from the effects of phase-induced intensity noise (PIIN) in addition to multiple-access interference (MAI) [9]. PIIN severely degrades the system performance and limits the number of simultaneous users. Several attempts to mitigate the effects of PIIN have appeared in literature [9]-[13]. Recently, we have proposed a very efficient method to come across the limitations due to PIIN [14]. In this method, we assign orthogonal multi-code sequences, from a minimum cross-correlation code set, to each user and encode the data symbols from each user using a multi-level $M$-ary pulse-position modulation ( $M$-PPM) scheme. During modulation, each level corresponds to a different code sequence, which would alleviate increasing the system bandwidth when using $M$-PPM. One possible code set that possesses both minimum cross-correlation and some orthogonal sequences is the modified prime sequence code [15].

On the other hand, it has been shown in [16] that low-complexity and cost-effective optical systems impose several important constraints on the signaling design. One of such constraints is the limited bandwidth due to impairments in electrooptic devices (e.g., electrooptic modulators) [17], modal dispersion in short-haul optical fiber links [18], and multipath distortion in diffuse indoor wireless optical links [19]. Furthermore, limited bandwidth of the optical devices imposes a constraint on the pulsewidth of the transmitted optical signal. Indeed, if $B_{s}$ denotes the system bandwidth, then the transmitted optical pulsewidth must be greater than $1 / B_{s}$. The limited bandwidths of the electrooptic modulator and other devices in the system (or equivalently the optical pulsewidth constraint) would raise another difficultly in practice. Indeed, this would limit the transmission rate of each user. Increasing the transmission rate (or throughput) without affecting the bandwidth is thus an ultimate goal in system design. Of course in this case the spectral efficiency would increase accordingly.

Under system bandwidth constraint (or optical pulsewidth constraint), the proposed technique in [14], to mitigate PIIN, 


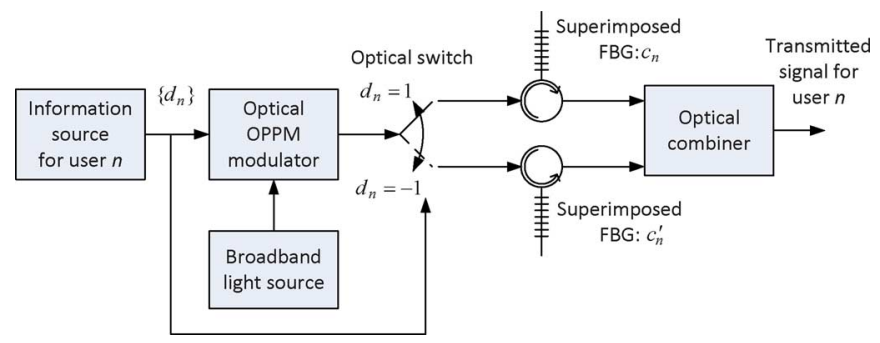

Fig. 1. A block diagram of a bipolar $M$-OPPM SAC-OCDMA transmitter for user $n \in\{1,2, \ldots, N\}$.

would only over-improve the bit-error rate (BER) performance. One would prefer to increase the transmission rate of a user and sacrifice some of its over-improved performance. To come across this stipulation we propose in this paper utilizing $M$-ary overlapping pulse-position modulation ( $M$-OPPM) scheme, rather than $M$-PPM scheme, in the system of [14]. OPPM technique has been first introduced by Lee and Schroeder [20] and further explored by a few other authors [21]-[24]. It has been shown that OPPM scheme offers a higher optical channel capacity, than traditional PPM does, without the need to reduce the light pulsewidth. Moreover, it has been noted in [21] that most of the capacity gain is achieved at a small overlap between light pulses. In addition, OPPM retains some of the advantages of PPM schemes over on-off keying (OOK) schemes. Namely, its transmitter utilizes the optical energy more efficiently and its receiver does not require a knowledge of the signal or noise power.

Furthermore, in this paper we analyze the performance of the proposed system and derive an expression for the bit error rate (BER), taking into account the effects of PIIN, shot noise, and thermal noise in addition to the MAI. Next we numerically compare the BER performance of this system to that adopting $M$-PPM or OOK schemes under a constraint on the optical pulsewidth. Our results reveal that under pulsewidth constraint, the proposed $M$-OPPM SAC-OCDMA system achieves acceptable performance with higher transmission rate, whereas $M$-PPM SAC-OCDMA achieves over-improved performance with lower transmission rate. In addition, the performance of proposed system is even better than that adopting OOK scheme with certain values of index of overlap.

The remaining of this paper is organized as follows. The system description and the receiver model are presented in Section II. Section III is devoted for the derivation of the bit error probabilities of the proposed system, taking into account the effects of several noise sources and MAI. In Section IV we present some numerical results, where we investigate the effect of some design parameters on the performance of the proposed scheme. Comparisons with corresponding systems adopting $M$-PPM and OOK techniques are also presented in same section. Finally, we give our conclusions in Section V.

\section{SYSTEM DESCRIPTION}

In this section we describe the system model including both the transmitter and receiver. In addition, we introduce the decision rule for the system.

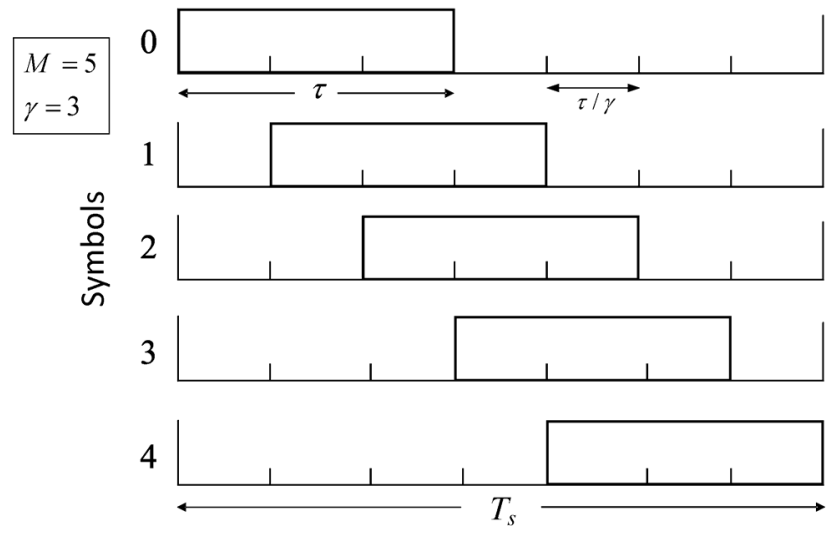

Fig. 2. An example of OPPM signal formats of a single user with $M=5$ and $\gamma=3$.

\section{A. Transmitter Side}

We consider a synchronous SAC-OCDMA network which is composed of $N$ users (or information sources). The $n$th information source, $n \in\{1,2, \ldots, N\}$, generates continuous iid $M$-ary data symbols or vectors $\left\{\boldsymbol{d}_{\boldsymbol{n}}\right\}$, where $\boldsymbol{d}_{\boldsymbol{n}}=\left[d_{n 0}, d_{n 1}, \ldots, d_{n, M-1}\right]$ such that $d_{n m} \in\{-1,1\}$ for some $m \in \mathcal{M} \stackrel{\text { def }}{=}\{0,1, \ldots, M-1\}$ and $d_{n l}=0$ for any other $l \in \mathcal{M}-\{m\}$. Fig. 1 shows the block diagram of the transmitter for user $n$. The generated sequence of symbols modulates the position of incoherent light pulses, emitted from an ideal broadband light source. Since there is a constraint on the pulsewidth, denoted by $\tau$, we adopt overlapping pulse-position modulation technique to allow for higher transmission rates. A light pulse is generated in slot $m \in \mathcal{M}$, out of $M$ possible overlapped slots, if $d_{n m} \neq 0$. The symbol duration, composed of $M$ overlapped slots, is denoted by $T_{s}$. In addition, each user is assigned two orthogonal code sequences of length $L=p^{2}$ and weight $w=p$, selected from same group of a modified-prime code set $\mathcal{C}(L, w, \lambda)$, where $p$ is a prime number and $\lambda=1$ is the maximum cross-correlation of the code. For example, user $n, n \in\{1,2, \ldots, N\}$, is assigned code sequences $\boldsymbol{c}_{\boldsymbol{n}}=\left\{c_{n}(1), c_{n}(2), \ldots, c_{n}(L)\right\}$ and $c_{\boldsymbol{n}}^{\prime}=\left\{c_{n}^{\prime}(1), c_{n}^{\prime}(2), \ldots, c_{n}^{\prime}(L)\right\}$, where $c_{n}(i), c_{n}^{\prime}(i) \in\{0,1\}$ and $c_{n}(i) c_{n}^{\prime}(i)=0$ for any $i \in\{1,2, \ldots, L\}$. Each code sequence is used to modulate the intensities of the spectral components of each emitted light pulse [7]-[11]. The modulation procedure for user $n$ is as follows: If for any $m \in \mathcal{M}$, $d_{n m}=1$, spectral code sequence $c_{\boldsymbol{n}}$ is used for modulation. If $d_{n m}=-1$, spectral code sequence $\boldsymbol{c}_{\boldsymbol{n}}^{\prime}$ is used, Fig. 1. Otherwise, no pulse is transmitted. Since both positive and negative data are considered, we call this technique bipolar M-OPPM $S A C-O C D M A$. In our analysis below, we denote the optical source frequency and bandwidth by $f_{c}$ and $\Delta \nu$, respectively. In addition, for mathematical convenience, we define the following two data variables for any user $n \in\{1,2, \ldots, N\}$ and slot $m \in \mathcal{M}$ :

$$
\alpha_{n m} \stackrel{\text { def }}{=} \frac{\left|d_{n m}\right|+d_{n m}}{2} \text { and } \beta_{n m} \stackrel{\text { def }}{=} \frac{\left|d_{n m}\right|-d_{n m}}{2} .
$$

Notice that $\alpha_{n m}, \beta_{n m} \in\{0,1\}$. Also it is clear that $\alpha_{n m} \cdot \beta_{n m}=$ $0, \alpha_{n m}+\beta_{n m}=\left|d_{n m}\right|$, and $\alpha_{n m}-\beta_{n m}=d_{n m}$. 


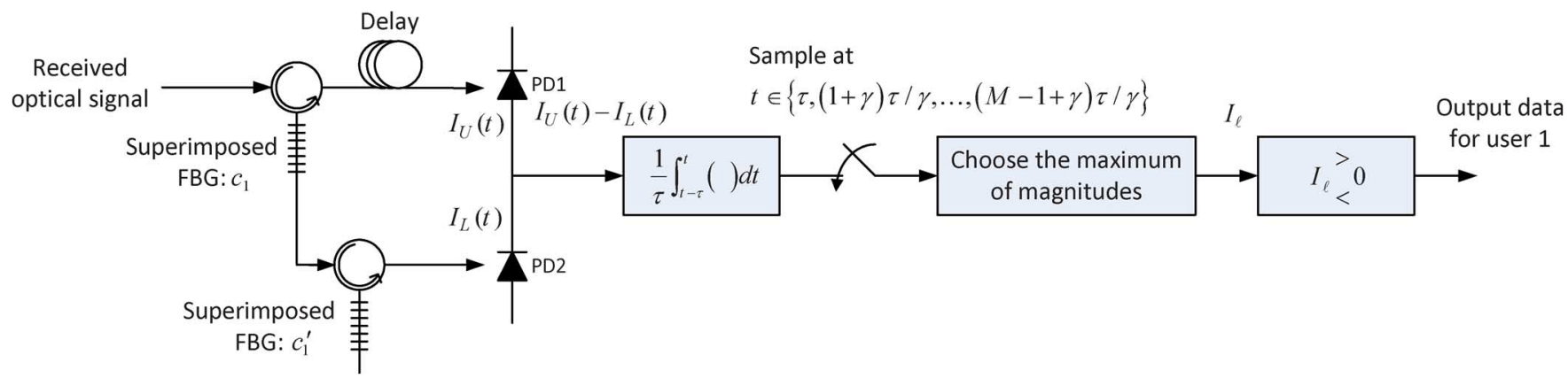

Fig. 3. A block diagram of a bipolar $M$-OPPM SAC-OCDMA receiver for user 1 .

\section{B. M-OPPM Technique and Data Transmission Rate}

In optical OPPM technique, with multiplicity $M$ and index of overlap $\gamma \in\{1,2, \ldots\}$, the information is conveyed by the position of a light pulse of duration $\tau$ within a time frame of width $T_{s}$. An overlap with depth $(1-1 / \gamma) \tau$ is allowed between any two adjacent positions. The transmitted pulse is said to be in position $x, x \in \mathcal{M}$, if it extends over the interval starting at time $x \tau / \gamma$ and ending $\tau$ s later, Fig. 2. This interval is also called a slot. It is obvious that each slot is subdivided into $\gamma$ smaller subintervals of width $\tau / \gamma$ and the relation between $T_{s}, \gamma, M$, and $\tau$ is given by:

$$
T_{s}=(M-1+\gamma) \frac{\tau}{\gamma}
$$

Assuming equi-probable data symbols, each is occurring with probability $1 / 2 M$, then the nominal transmission rate is given by:

$$
R_{b}=\frac{\log _{2}(2 M)}{T_{s}}=\frac{\gamma \log _{2}(2 M)}{(M-1+\gamma) \tau} \quad \mathrm{bit} / \mathrm{s} .
$$

It should be noticed that under pulsewidth constraint, the transmission rates of both on-off keying (OOK) and bipolar 2-PPM are equal to $1 / \tau$. It is also remarkable that PPM can be considered as a special case of OPPM when $\gamma=1$.

\section{Code Properties and Number of Users}

The cross-correlation function between any two code sequences $n, m \in \mathcal{C}(L, w, \lambda)$ is given by [15]

$$
\begin{aligned}
& \sum_{i=1}^{L} c_{n}(i) c_{m}(i) \\
& \quad= \begin{cases}p ; & \text { if } n=m, \\
0 ; & \text { if } n \text { and } m \text { share the same group and } n \neq m, \\
1 ; & \text { if } n \text { and } m \text { are from different groups. }\end{cases}
\end{aligned}
$$

Since the total number of available code sequences in any group of $\mathcal{C}(L, w, \lambda)$ is $p$ and each user is assigned two code sequences, the total number of network subscribers cannot exceed $p\lfloor p / 2\rfloor$, [14]. Here $\lfloor x\rfloor$ denotes the largest integer not greater than $x$. Out of this number we assume that there are $N$ active or simultaneous users and the remaining users are idle. Without loss of generality, we always assume that the first user is the desired user. Let the random variable $H$ represent the number of active users in the first group. For uniform code assignment, it has been shown in [14] that the probability distribution of $H$, given that user 1 is always active, can be written as

$$
P_{H}(h)=\frac{\left(\begin{array}{c}
\left\lfloor\frac{p}{2}\right\rfloor-1 \\
h-1
\end{array}\right)\left(\begin{array}{c}
(p-1)\left\lfloor\frac{p}{2}\right\rfloor \\
N-h
\end{array}\right)}{\left(\begin{array}{c}
p\left\lfloor\frac{p}{2}\right\rfloor-1 \\
N-1
\end{array}\right)},
$$

where $h \in\left\{h_{\min }, h_{\min }+1, \ldots, h_{\max }\right\}$ and

$$
\begin{aligned}
& h_{\min }=\max \left\{1, N-(p-1)\left\lfloor\frac{p}{2}\right\rfloor\right\} \\
& h_{\max }=\min \left\{N,\left\lfloor\frac{p}{2}\right\rfloor\right\} .
\end{aligned}
$$

\section{Receiver Side and Decision Rule}

The block diagram of the bipolar $M$-OPPM SAC-OCDMA receiver is shown in Fig. 3 for the desired user. It is pretty much similar to that used in $M$-PPM SAC-OCDMA system [14], but the sample instants are modified to:

$$
t \in\left\{\tau,(1+\gamma) \frac{\tau}{\gamma},(2+\gamma) \frac{\tau}{\gamma}, \ldots,(M-1+\gamma) \frac{\tau}{\gamma}\right\} .
$$

In this receiver, a balanced detection scheme is adopted, where the upper and lower branches are used to detect received spectral components that correspond to user's code sequences $\boldsymbol{c}_{\mathbf{1}}$ and $\boldsymbol{c}_{\mathbf{1}}^{\prime}$, respectively. In order to avoid delays between reflected spectral components, the code detections are implemented using superimposed fiber Bragg gratings (FBGs) [25], [26]. Both photodiodes' output currents are then subtracted, time averaged, and sampled at the above time instants. This produces a set of decision currents $\left\{I_{0}, I_{1}, \ldots, I_{M-1}\right\}$, which is finally passed to the decision circuit.

1) Decision Rule: The decision current $I_{\ell}$ over slot $\ell \in \mathcal{M}$ is defined as $I_{\ell}=I_{U, \ell}-I_{L, \ell}$, where $I_{U, \ell}$ and $I_{L, \ell}$ are the average output currents of the upper- and lower-branch photodiode, respectively. We define the decision rule as follows:

$$
\text { Decide } \begin{cases}\boldsymbol{b}^{\ell,+} ; & \text { if } I_{\ell}>\left|I_{m}\right| \quad \forall m \in \mathcal{M}-\{\ell\}, \\ \boldsymbol{b}^{\ell,-} ; & \text { if } I_{\ell}<-\left|I_{m}\right| \quad \forall m \in \mathcal{M}-\{\ell\},\end{cases}
$$

where $\boldsymbol{b}^{m, \pm}=\left[b_{0}^{m}, b_{1}^{m}, \ldots, b_{M-1}^{m}\right]$ with $b_{m}^{m}= \pm 1$ and $b_{i}^{m}=0$ for any $i \in \mathcal{M}-\{m\}$.

\section{TheORETICAL ANALYSIS}

In this section we derive the statistics of both upper- and lower-branch currents, as well as the statistics of the decision 
current. In addition, we develop an expression for evaluating the BER of the system.

\section{A. Statistics of Upper-Branch Current}

The resultant upper-branch incident field of the $\ell$ th slot, $\ell \in$ $\mathcal{M}$, can be written as:

$$
E_{U, \ell}(t)=\sqrt{\frac{P_{0}}{L}} \sum_{n=1}^{N} \sum_{i=1}^{L} \xi_{n, \ell}^{i}(t) c_{1}(i) e^{j\left[\omega_{0} t+i \delta \omega t+\phi_{n i}(t)\right]},
$$

where

$$
\begin{array}{r}
\xi_{n, \ell}^{i}(t)=\sum_{r=-\min \{\gamma-1, \ell\}}^{\min \{\gamma-1, M-1-\ell\}}\left[\alpha_{n, \ell+r} c_{n}(i)+\beta_{n, \ell+r} c_{n}^{\prime}(i)\right] \\
\times g\left[\frac{t-(\ell+r \vee 0) \frac{\tau}{\gamma}}{\left(1-\frac{|r|}{\gamma}\right) \tau}\right],
\end{array}
$$

$n \in\{1,2, \ldots, N\}, i \in\{1,2, \ldots, L\}$, is the response of user $n$ in slot $\ell$ due to its $i$ th spectral component, $P_{0}$ is the peak received single-user power, $\delta \omega=2 \pi \Delta \nu / L, \omega_{0}=2 \pi\left(f_{c}-\right.$ $\Delta \nu / 2-\Delta \nu / L), g(t)$ is a normalized envelope of a single-user light field of spectral width $\Delta \nu / L$, and $\phi_{n i}(t)$ is the random phase of the $i$ th chip optical signal of the $n$th user, assumed to be a Wiener process [27]-[29]. Here $x \vee y \stackrel{\text { def }}{=} \max \{x, y\}$. For the sake of simplicity, we assume a rectangular envelope:

$$
g(t)= \begin{cases}1 ; & \text { if } t \in[0,1] \\ 0 ; & \text { otherwise }\end{cases}
$$

It should be noticed that there is at most one nonzero term in the summation in (9). If the photodiode responsivity is denoted by $\mathcal{R}$, then the average $\ell$ th slot current of upper photodiode PD1 is given by

$$
I_{U, \ell}=\frac{\mathcal{R}}{\tau} \int_{\frac{\ell \tau}{\gamma}}^{(\ell / \gamma+1) \tau}\left|E_{U, \ell}(t)\right|^{2} d t
$$

Using (8) and (11), and noticing that the cross-terms cancel out because of the averaging process (as long as $\tau>L / \Delta \nu$ ), we get:

$$
I_{U, \ell}=\frac{\mathcal{R} P_{0}}{L} \sum_{i=1}^{L} \frac{1}{\tau} \times \int_{\frac{\ell \tau}{\gamma}}^{(\ell / \gamma+1) \tau}\left|\sum_{n=1}^{N} \xi_{n, \ell}^{i}(t) c_{1}(i) e^{j \phi_{n i}(t)}\right|^{2} d t .
$$

Expanding the last equation, we get

$$
\begin{aligned}
I_{U, \ell}=\frac{\mathcal{R} P_{0}}{L} \sum_{i=1}^{L}[ & \sum_{n=1}^{N} c_{1}(i) \frac{1}{\tau} \int_{\frac{\ell \tau}{\gamma}}^{(\ell / \gamma+1) \tau} \xi_{n, \ell}^{i}(t) d t \\
& +2 \sum_{n=1}^{N-1} \sum_{m=n+1}^{N} c_{1}(i) \frac{1}{\tau} \int_{\frac{\ell \tau}{\gamma}}^{(\ell / \gamma+1) \tau} \xi_{n, \ell}^{i}(t) \xi_{m, \ell}^{i}(t) \\
& \left.\times \cos \left(\phi_{n i}(t)-\phi_{m i}(t)\right) d t\right]
\end{aligned}
$$

For convenience, we define $r_{\min }(\ell)$ and $r_{\max }(\ell)$, for any $\ell \in$ $\mathcal{M}$, as follows:

$$
\begin{aligned}
& r_{\min }(\ell) \stackrel{\text { def }}{=}-\min \{\gamma-1, \ell\} \\
& r_{\max }(\ell) \stackrel{\text { def }}{=} \min \{\gamma-1, M-1-\ell\},
\end{aligned}
$$

respectively. Noticing that

$$
\begin{aligned}
& \frac{1}{\tau} \int_{\frac{\ell \tau}{\gamma}}^{(\ell / \gamma+1) \tau} \xi_{n, \ell}^{i}(t) d t \\
& =\sum_{r=r_{\min }(\ell)}^{r_{\text {max }}(\ell)}\left[\alpha_{n, \ell+r} c_{n}(i)+\beta_{n, \ell+r} c_{n}^{\prime}(i)\right] \\
& \quad \times \frac{1}{\tau} \int_{r=r_{\min }(\ell)}^{(\ell / \gamma+1) \tau}\left[\frac{t-(\ell+r \vee 0) \frac{\tau}{\gamma}}{\left(1-\frac{|r|}{\gamma}\right) \tau}\right] d t \\
& =\sum_{r_{\text {max }}(\ell)}^{\frac{\ell \tau}{\gamma}}\left[\alpha_{n, \ell+r} c_{n}(i)+\beta_{n, \ell+r} c_{n}^{\prime}(i)\right]\left(1-\frac{|r|}{\gamma}\right)
\end{aligned}
$$

we get

$$
\begin{aligned}
I_{U, \ell}=\frac{\mathcal{R} P_{0}}{L} \sum_{i=1}^{L}\{ & \sum_{n=1}^{N} c_{1}(i) \\
& \times \sum_{r=r_{\min }(\ell)}^{r_{\max }(\ell)} a_{r}\left[\alpha_{n, \ell+r} c_{n}(i)+\beta_{n, \ell+r} c_{n}^{\prime}(i)\right] \\
& +2 \sum_{n=1}^{N-1} \sum_{m=n+1}^{N} c_{1}(i) \frac{1}{\tau} \\
& \times \int_{(\ell / \gamma+1) \tau}^{\frac{\ell \tau}{\gamma}} \xi_{n, \ell}^{i}(t) \xi_{m, \ell}^{i}(t) \\
& \left.\times \cos \left(\phi_{n i}(t)-\phi_{m i}(t)\right) d t\right\}
\end{aligned}
$$

where

$$
a_{r} \stackrel{\text { def }}{=}\left(1-\frac{|r|}{\gamma}\right)
$$

Making use of the properties of $\mathcal{C}(L, w, \lambda)$ code and assuming that the number of active users in group 1 is $h$, we get

$$
\begin{aligned}
I_{U, \ell}=\frac{\mathcal{R} P_{0}}{L}[w & \sum_{r=r_{\min }(\ell)}^{r_{\max }(\ell)} a_{r} \alpha_{1, \ell+r}+\lambda \sum_{r=r_{\min }(\ell)}^{r_{\max }(\ell)} a_{r} \kappa_{\ell+r} \\
& +2 \sum_{n=1}^{N-1} \sum_{m=n+1}^{N} \sum_{i=1}^{L} c_{1}(i) \frac{1}{\tau} \int_{\frac{\ell \tau}{\gamma}}^{(\ell / \gamma+1) \tau} \xi_{n, \ell}^{i}(t) \xi_{m, \ell}^{i}(t) \\
& \left.\times \cos \left(\phi_{n i}(t)-\phi_{m i}(t)\right) d t\right] .
\end{aligned}
$$


Here we defined the interference random variable $\kappa_{m}$ for any $m \in \mathcal{M}$ as:

$$
\kappa_{m} \stackrel{\text { def }}{=} \sum_{n=h+1}^{N}\left|d_{n m}\right|
$$

In addition, we define the interference random vector $\boldsymbol{\kappa} \stackrel{\text { def }}{=}$ $\left[\kappa_{0}, \kappa_{1}, \ldots, \kappa_{M-1}\right]$. Of course $\kappa_{m}$ follows a conditional binomial distribution and $\boldsymbol{\kappa}$ follows a conditional multinomial distribution, given $H=h$ :

$$
\begin{aligned}
P_{\kappa_{m} \mid H}\left(l_{m} \mid h\right) & =\left(\begin{array}{c}
N-h \\
l_{m}
\end{array}\right)\left(\frac{1}{M}\right)^{l_{m}}\left(1-\frac{1}{M}\right)^{N-h-l_{m}} \\
P_{\boldsymbol{\kappa} \mid H}(\boldsymbol{l} \mid h) & =\frac{(N-h) !}{l_{0} ! l_{1} ! l_{2} ! \ldots l_{M-1} !}\left(\frac{1}{M}\right)^{N-h}
\end{aligned}
$$

where $l_{m} \in\{0,1, \ldots, N-h\}$ and $\boldsymbol{l} \stackrel{\text { def }}{=}\left[l_{0}, l_{1}, \ldots, l_{M-1}\right]$, such that $\sum_{m=0}^{M-1} l_{m}=N-h$.

Now we consider the last integration in (18). In order to perform the product of the two $\xi$ functions, first it is easy to check the following product:

$$
\begin{gathered}
g\left[\frac{t-(\ell+r \vee 0) \frac{\tau}{\gamma}}{\left(1-\frac{|r|}{\gamma}\right) \tau}\right] g\left[\frac{t-(\ell+s \vee 0) \frac{\tau}{\gamma}}{\left(1-\frac{|s|}{\gamma}\right) \tau}\right] \\
=g\left[\frac{t-(\ell+r \vee s \vee 0) \frac{\tau}{\gamma}}{\left(1-\frac{1}{\gamma} \max \{|r|,|s|,|r-s| \wedge \gamma\}\right) \tau}\right],
\end{gathered}
$$

where $x \wedge y \stackrel{\text { def }}{=} \min \{x, y\}$. In addition, we define the following random variable for any $i \in\{1,2, \ldots, L\}$ and any $n, m \in$ $\{1,2, \ldots, N\}$ with $n \neq m$ :

$X_{n m}^{i}(\ell, r, s) \stackrel{\text { def }}{=} \frac{1}{\tau} \int_{\frac{\ell \tau}{\gamma}}^{(\ell / \gamma+1) \tau} g\left(\frac{t-t_{0}}{b_{r s} \tau}\right) \times \cos \left(\phi_{n i}(t)-\phi_{m i}(t)\right) d t$,

where $t_{0}=(\ell+r \vee s \vee 0) \tau / \gamma$ and $b_{r s}=1-$ $(1 / \gamma) \max \{|r|,|s|,|r-s| \wedge \gamma\}$. Substituting back in (18), we get

$$
\begin{aligned}
I_{U, \ell}=\frac{\mathcal{R} P_{0}}{L}[ & w \sum_{r=r_{\min }(\ell)}^{r_{\max }(\ell)} a_{r} \alpha_{1, \ell+r}+\lambda \sum_{r=r_{\min }(\ell)}^{r_{\max }(\ell)} a_{r} \kappa_{\ell+r} \\
& +2 \sum_{n=1}^{N-1} \sum_{m=n+1}^{N} \sum_{i=1}^{L} c_{1}(i) \\
& \times \sum_{r=r_{\min }(\ell)}^{r_{\max }(\ell)} \sum_{s=r_{\min }(\ell)}^{r_{\max }(\ell)}\left[\alpha_{n, \ell+r} c_{n}(i)+\beta_{n, \ell+r} c_{n}^{\prime}(i)\right] \\
& \left.\times\left[\alpha_{m, \ell+s} c_{m}(i)+\beta_{m, \ell+s} c_{m}^{\prime}(i)\right] X_{n m}^{i}(\ell, r, s)\right]
\end{aligned}
$$

Using [27]-[29], the average and variance of $X_{n m}^{i}(\ell, r, s)$ are

$$
\begin{aligned}
& \mu_{X_{n m}^{i}(\ell, r, s)} \\
& \stackrel{\text { def }}{=} E\left\{X_{n m}^{i}(\ell, r, s)\right\}=0 \\
& \sigma_{X_{n m}^{i}(\ell, r, s)}^{2} \\
& \stackrel{\text { def }}{=} \operatorname{var}\left\{X_{n m}^{i}(\ell, r, s)\right\} \\
& =\frac{1}{2 \tau^{2}} \int_{\frac{\ell \tau}{\gamma}}^{(\ell / \gamma+1) \tau} \int_{\frac{\ell \tau}{\gamma}}^{(\ell / \gamma+1) \tau} g^{2}\left(\frac{t-t_{0}}{b_{r s} \tau}\right) \\
& \times g^{2}\left(\frac{v-t_{0}}{b_{r s} \tau}\right) e^{-2 \frac{|t-v|}{\tau_{c}}} d t d v,
\end{aligned}
$$

respectively, where $\tau_{c}$ is the coherence time. Performing the last integration, we obtain

$$
\begin{aligned}
\sigma_{X_{n m}^{i}(\ell, r, s)}^{2} & =\frac{\tau_{c}}{2 \tau}\left[b_{r s}-\frac{\tau_{c}}{2 \tau}\left(1-e^{-2 \frac{b_{r s} \tau}{\tau_{c}}}\right)\right] \\
& \approx b_{r s} \frac{\tau_{c}}{2 \tau}=b_{r s} B_{e} \tau_{c},
\end{aligned}
$$

where $B_{e}$ is the receiver electrical bandwidth and we have used the practical assumption that $\tau_{c} \ll 1 /\left(\gamma B_{e}\right)$. To get the average and variance of $I_{U, \ell}$, we use (24) and (25) in (23), taking into account the independence of the random variables $X_{n m}^{i}(\ell, r, s)$ :

$$
\begin{aligned}
& \mu_{I_{U} \mid \ell, \boldsymbol{d}_{1}, \kappa} \\
& \stackrel{\text { def }}{=} E\left\{I_{U, \ell}\right\} \\
& =\frac{\mathcal{R} P_{0}}{L}\left[w \sum_{r=r_{\min }(\ell)}^{r_{\max }(\ell)} a_{r} \alpha_{1, \ell+r}+\lambda \sum_{r=r_{\min }(\ell)}^{r_{\max }(\ell)} a_{r} \kappa_{\ell+r}\right] \\
& \sigma_{I_{U} \mid \ell, \boldsymbol{d}_{1, \boldsymbol{\kappa}}}^{2} \\
& \stackrel{\text { def }}{=} \operatorname{var}\left\{I_{U, \ell}\right\} \\
& =4 B_{e} \tau_{c}\left(\frac{\mathcal{R} P_{0}}{L}\right)^{2} \\
& \times\left[\sum_{n=1}^{N-1} \sum_{m=n+1}^{N} \sum_{i=1}^{L} c_{1}(i)\right. \\
& \times \sum_{r=r_{\min }(\ell)}^{r_{\max }(\ell)} \sum_{s=r_{\min }(\ell)}^{r_{\max }(\ell)}\left[\alpha_{n, \ell+r} c_{n}(i)+\beta_{n, \ell+r} c_{n}^{\prime}(i)\right] \\
& \left.\times\left[\alpha_{m, \ell+s} c_{m}(i)+\beta_{m, \ell+s} c_{m}^{\prime}(i)\right] b_{r s}\right]
\end{aligned}
$$

Making use of the properties of $\mathcal{C}(L, w, \lambda)$ code and noticing that the average of the sum of products of three different codes is equal to $\lambda w / L$, the last variance becomes:

$$
\begin{aligned}
& \sigma_{I_{U} \mid \ell, \boldsymbol{d}_{1}, \boldsymbol{\kappa}}^{2} \\
& =4 B_{e} \tau_{c}\left(\frac{\mathcal{R} P_{0}}{L}\right)^{2}\left[\lambda \sum_{r=r_{\min }(\ell)}^{r_{\max }(\ell)} \sum_{s=r_{\min }(\ell)}^{r_{\max }(\ell)} b_{r s} \alpha_{1, \ell+r} \kappa_{\ell+s}\right.
\end{aligned}
$$




$$
\begin{aligned}
& +\frac{\lambda w}{L} \sum_{r=r_{\min }(\ell)}^{r_{\max }(\ell)} \sum_{s=r_{\min }(\ell)}^{r_{\max }(\ell)} b_{r s} \\
& \left.\times \sum_{n=h+1}^{N-1} \sum_{m=n+1}^{N}\left|d_{n, \ell+r}\right|\left|d_{m, \ell+s}\right|\right] \\
=4 B_{e} \tau_{c}\left(\frac{\mathcal{R} P_{0}}{L}\right)^{2}[ & \sum_{r=r_{\min }(\ell)}^{r_{\text {max }}(\ell)} \sum_{s=r_{\min }(\ell)}^{r_{\text {max }}(\ell)} b_{r s} \\
& \times\left(\lambda \alpha_{1, \ell+r}+\frac{\lambda w}{L} \cdot \frac{\kappa_{\ell+r}}{2}\right) \kappa_{\ell+s} \\
& \left.-\sum_{r=r_{\min }(\ell)}^{r_{\max }(\ell)} a_{r} \frac{\lambda w}{L} \cdot \frac{\kappa_{\ell+r}}{2}\right] .
\end{aligned}
$$

\section{B. Statistics of Lower-Branch Current}

The resultant lower-branch incident field of the $\ell$ th slot, $\ell \in$ $\mathcal{M}$, can be written as:

$$
E_{L, \ell}(t)=\sqrt{\frac{P_{0}}{L}} \sum_{n=1}^{N} \sum_{i=1}^{L} \xi_{n, \ell}^{i}(t) c_{1}^{\prime}(i) e^{j\left[\omega_{0} t+i \delta \omega t+\phi_{n i}(t)\right]} .
$$

It should be noticed that $E_{L, \ell}(t)$ is independent of $E_{U, \ell}(t)$, as the chips selected by $\left\{c_{1}^{\prime}(i)\right\}$ and $\left\{c_{1}(i)\right\}$ are disjoint. Following a similar analysis to what we did in the last subsection, we get the average and variance of lower-branch average $\ell$ th slot current $I_{L, \ell}$ as:

$$
\begin{aligned}
& \mu_{I_{L} \mid \ell, \boldsymbol{d}_{1}, \kappa} \\
& \stackrel{\text { def }}{=} E\left\{I_{L, \ell}\right\} \\
& =\frac{\mathcal{R} P_{0}}{L}\left[w \sum_{r=r_{\min }(\ell)}^{r_{\max }(\ell)} a_{r} \beta_{1, \ell+r}+\lambda \sum_{r=r_{\min }(\ell)}^{r_{\max }(\ell)} a_{r} \kappa_{\ell+r}\right] \\
& \sigma_{I_{L} \mid \ell, \boldsymbol{d}_{1}, \kappa}^{2} \\
& \stackrel{\text { def }}{=} \operatorname{var}\left\{I_{L, \ell}\right\} \\
& =4 B_{e} \tau_{c}\left(\frac{\mathcal{R} P_{0}}{L}\right)^{2}\left[\sum_{r=r_{\min }(\ell)}^{r_{\max }(\ell)} \sum_{s=r_{\min }(\ell)}^{r_{\max }(\ell)} b_{r s}\right. \\
& \times\left(\lambda \beta_{1, \ell+r}+\frac{\lambda w}{L} \cdot \frac{\kappa_{\ell+r}}{2}\right) \kappa_{\ell+s} \\
& \left.-\sum_{r=r_{\min }(\ell)}^{r_{\max }(\ell)} a_{r} \frac{\lambda w}{L} \cdot \frac{\kappa_{\ell+r}}{2}\right] \text {, }
\end{aligned}
$$

respectively.

\section{Statistics of Decision Current}

From the discussion in the above subsection, the decision current $I_{\ell}=I_{U, \ell}-I_{L, \ell}$ over slot $\ell \in \mathcal{M}$, has the following mean and variance, given desired user data vector $\boldsymbol{d}_{\mathbf{1}}=\boldsymbol{b}=\left[b_{0}, b_{1}, \ldots, b_{M-1}\right]$ and interference vector $\boldsymbol{\kappa}=\boldsymbol{l}=\left[l_{0}, l_{1}, \ldots, l_{M-1}\right]$,

$$
\begin{aligned}
\mu_{I}(\ell, \boldsymbol{b}) & =\frac{\mathcal{R} P_{0}}{L} w \sum_{r=r_{\min }(\ell)}^{r_{\max }(\ell)} a_{r} b_{\ell+r} \\
\sigma_{I}^{2}(\ell, \boldsymbol{b}, \boldsymbol{l}) & =\sigma_{P I I N \mid \ell, \boldsymbol{b}, \boldsymbol{l}}^{2}+\sigma_{s \mid \ell, \boldsymbol{b}, \boldsymbol{l}}^{2}+\sigma_{T}^{2},
\end{aligned}
$$

respectively, where $\sigma_{P I I N \mid \ell, \boldsymbol{b}, \boldsymbol{l}}^{2}, \sigma_{s \mid \ell, \boldsymbol{b}, \boldsymbol{l}}^{2}$, and $\sigma_{T}^{2}$ are the variances of the phase-induced intensity noise, shot noise, and thermal noise, respectively. These are given by

$$
\begin{aligned}
& \sigma_{P I I N \mid \ell, \boldsymbol{b}, \boldsymbol{l}}^{2} \\
& =\sigma_{I_{U} \mid \ell, \boldsymbol{b}, \boldsymbol{l}}^{2}+\sigma_{I_{L} \mid \ell, \boldsymbol{b}, \boldsymbol{l}}^{2} \\
& =4 B_{e} \tau_{c}\left(\frac{\mathcal{R} P_{0}}{L}\right)^{2}\left[\sum_{r=r_{\min }(\ell)}^{r_{\max }(\ell)} \sum_{s=r_{\min }(\ell)}^{r_{\max }(\ell)} b_{r s}\right. \\
& \times\left(\lambda\left|b_{\ell+r}\right|+\frac{\lambda w}{L} \cdot l_{\ell+r}\right) l_{\ell+s} \\
& \left.-\sum_{r=r_{\min }(\ell)}^{r_{\max }(\ell)} a_{r} \frac{\lambda w}{L} \cdot l_{\ell+r}\right] \\
& \sigma_{s \mid \ell, \boldsymbol{b}, \boldsymbol{l}}^{2}=2 e B_{e}\left(\mu_{I_{U} \mid \ell, \boldsymbol{b}, \boldsymbol{l}}+\mu_{I_{L} \mid \ell, \boldsymbol{b}, \boldsymbol{l}}\right) \\
& =2 e B_{e} \frac{\mathcal{R} P_{0}}{L}\left[\sum_{r=r_{\min }(\ell)}^{r_{\max }(\ell)} a_{r}\left(w\left|b_{\ell+r}\right|+2 \lambda l_{\ell+r}\right)\right] \\
& \sigma_{T}^{2}=\frac{4 k_{B} T^{\circ} B_{e}}{R_{L}},
\end{aligned}
$$

respectively. Here $e=1.6 \times 10^{-19} \mathrm{C}$ is the electron charge, $k_{B}=1.38 \times 10^{-23} \mathrm{~J} / \mathrm{K}$ is Boltzmann's constant, $T^{\circ}$ is the receiver noise temperature, and $R_{L}$ is the receiver load resistance.

\section{BER Evaluation}

The average symbol error rate (SER) $P_{e}$ can be written as

$$
\begin{aligned}
P_{e}=\frac{1}{2 M} \sum_{h=h_{\min }}^{h_{\max }} \sum_{\boldsymbol{l} \in \mathcal{X}} \sum_{m=0}^{M-1}\left[P_{e \mid \boldsymbol{d}_{1}, \boldsymbol{\kappa}, H}\left(\boldsymbol{b}^{m,+}, \boldsymbol{l}, h\right)\right. \\
\left.\quad+P_{e \mid \boldsymbol{d}_{1}, \boldsymbol{\kappa}, H}\left(\boldsymbol{b}^{m,-}, \boldsymbol{l}, h\right)\right] P_{\boldsymbol{\kappa} \mid H}(\boldsymbol{l} \mid h) P_{H}(h),
\end{aligned}
$$

where the set $\mathcal{X} \stackrel{\text { def }}{=}\left\{\boldsymbol{l}=\left[l_{0}, l_{1}, \ldots, l_{M-1}\right]: \sum_{m=0}^{M-1} l_{m}=\right.$ $N-h\}, P_{e \mid \boldsymbol{d}_{1}, \boldsymbol{\kappa}, H}\left(\boldsymbol{b}^{m, \pm}, \boldsymbol{l}, h\right)$ is the conditional SER given a transmitted data symbol $\boldsymbol{d}_{\mathbf{1}}=\boldsymbol{b}^{m, \pm}$, an interference vector $\boldsymbol{\kappa}=\boldsymbol{l}$, and $H=h$ users in first group. Here $\boldsymbol{b}^{m, \pm}=\left[b_{0}^{m}, b_{1}^{m}, \ldots, b_{M-1}^{m}\right]$ with $b_{m}^{m}= \pm 1$ and $b_{i}^{m}=0$ for any $i \in \mathcal{M}-\{m\}$. Because of the symmetry of the bipolar modulation format, the last equation reduces to:

$$
\begin{array}{r}
P_{e}=\frac{1}{M} \sum_{h=h_{\text {min }}}^{h_{\text {max }}} \sum_{\boldsymbol{l} \in \mathcal{X}} \sum_{m=0}^{M-1} P_{e \mid \boldsymbol{d}_{1}, \boldsymbol{\kappa}, H}\left(\boldsymbol{b}^{m,+}, \boldsymbol{l}, h\right) \\
\times P_{\boldsymbol{\kappa} \mid H}(\boldsymbol{l} \mid h) P_{H}(h),
\end{array}
$$


Using a union bound, the last conditional SER can be upperbounded as:

$$
\begin{aligned}
& P_{e \mid \boldsymbol{d}_{\mathbf{1}}, \boldsymbol{\kappa}, H}\left(\boldsymbol{b}^{m,+}, \boldsymbol{l}, h\right) \\
& =\operatorname{Pr}\left\{I_{m} \leq\left|I_{\ell}\right|, \text { some } \ell \in \mathcal{M}-\{m\} \mid \boldsymbol{d}_{\mathbf{1}}=\boldsymbol{b}^{m,+},\right. \\
& \leq \sum_{u=-m, u \neq 0}^{\boldsymbol{\kappa}=\boldsymbol{l}, H=h\}} \operatorname{Pr}\left\{I_{m} \leq\left|I_{m+u}\right| \mid \boldsymbol{d}_{\mathbf{1}}=\boldsymbol{b}^{m,+}, \boldsymbol{\kappa}=\boldsymbol{l}, H=h\right\} \\
& \leq \sum_{u=-m, u \neq 0}^{M-1-m}\left[\operatorname{Pr}\left\{I_{m} \leq I_{m+u} \mid \boldsymbol{d}_{\mathbf{1}}=\boldsymbol{b}^{m,+}, \boldsymbol{\kappa}=\boldsymbol{l}, H=h\right\}\right. \\
& =2 \sum_{u=-m, u \neq 0}^{M-1-m} \operatorname{Pr}\left\{I_{m} \leq I_{m+u} \mid \boldsymbol{d}_{\mathbf{1}}=\boldsymbol{b}^{m,+},\right. \\
& \quad \boldsymbol{\kappa}=\boldsymbol{l}, H=h\},
\end{aligned}
$$

where the last equality is justified by noticing that the mean of $I_{m+u}$, given $\boldsymbol{d}_{\mathbf{1}}=\boldsymbol{b}^{m,+}$, is zero for $u \neq 0$. Substituting in (33), we get

$$
\begin{aligned}
& P_{e} \leq \frac{2}{M} \sum_{h=h_{\min }}^{h_{\max }} \sum_{\boldsymbol{l} \in \mathcal{X}} P_{\boldsymbol{\kappa} \mid H}(\boldsymbol{l} \mid h) P_{H}(h) \\
& \times \sum_{m=0}^{M-1} \sum_{u=-m, u \neq 0}^{M-1-m} \operatorname{Pr}\left\{I_{m} \leq I_{m+u} \mid \boldsymbol{d}_{\mathbf{1}}=\boldsymbol{b}^{m,+},\right. \\
&\boldsymbol{\kappa}=\boldsymbol{l}, H=h\} \frac{4}{M} \sum_{h=h_{\min }}^{h_{\text {max }}} \sum_{\boldsymbol{l} \in \mathcal{X}} P_{\boldsymbol{\kappa} \mid H}(\boldsymbol{l} \mid h) P_{H}(h) \sum_{m=0}^{M-2} \\
& \times \sum_{u=1}^{M-1-m} \operatorname{Pr}\left\{I_{m} \leq I_{m+u} \mid \boldsymbol{d}_{\mathbf{1}}=\boldsymbol{b}^{m,+},\right. \\
&\boldsymbol{\kappa}=\boldsymbol{l}, H=h\} .
\end{aligned}
$$

The last summation is simplified as

$$
\begin{gathered}
\sum_{u=1}^{M-1-m} \operatorname{Pr}\left\{I_{m} \leq I_{m+u} \mid \boldsymbol{d}_{\mathbf{1}}=\boldsymbol{b}^{m,+}, \boldsymbol{\kappa}=\boldsymbol{l}, H=h\right\} \\
=\sum_{u=1}^{r_{\max }(m)} \operatorname{Pr}\left\{Y_{m} \leq Z_{m+u} \mid \boldsymbol{d}_{\mathbf{1}}=\boldsymbol{b}^{m,+}, \boldsymbol{\kappa}=\boldsymbol{l}, H=h\right\} \\
+\sum_{u=r_{\max }(m)+1}^{M-1-m} \operatorname{Pr}\left\{I_{m} \leq I_{m+u} \mid \boldsymbol{d}_{\mathbf{1}}=\boldsymbol{b}^{m,+},\right. \\
\boldsymbol{\kappa}=\boldsymbol{l}, H=h\},
\end{gathered}
$$

where $r_{\max }(m)$ was defined in (14) and the independent random variables $Y_{m}$ and $Z_{m}$ are defined in Appendix A. Using the Gaussian approximation method, the evaluations of the last two probabilities are immediate and the bit-error rate can be approximated as given in (37) at the bottom of the page, where $\mu_{Y}(\cdot)$, $\sigma_{Y}^{2}(\cdot), \mu_{Z}(\cdot)$, and $\sigma_{Z}^{2}(\cdot)$ are given in Appendix A. In is easy to verify that in the special case of $\gamma=1, M$-OPPM reduces to $M$-PPM and (37) reduces to the expression given in [14].

\section{Numerical Results}

In our numerical evaluations we assume that both the optical pulsewidth $\tau$ (or receiver bandwidth $B_{e}=1 / 2 \tau$ ) and the average transmitted photons per nat $\mu$ are held fixed. For an ideal channel, the peak single-user received power is given by:

$$
P_{0}= \begin{cases}2 \log 2\left(\frac{\eta e}{\mathcal{R}}\right) B_{e} \mu ; & \text { for Bipolar OOK, } \\ 2 \log (2 M)\left(\frac{\eta e}{\mathcal{R}}\right) B_{e} \mu ; & \text { for Bipolar } M-\mathrm{PPM}, \\ 2 \log (2 M)\left(\frac{\eta e}{\mathcal{R}}\right) B_{e} \mu ; & \text { for Bipolar } M-\mathrm{OPPM},\end{cases}
$$

where $\eta$ is the photodiode efficiency, assumed unity. The bit-error rate (BER) of the bipolar $M$-OPPM SAC-OCDMA system, as given in (37), has been evaluated numerically for a modified prime sequence (MPS) code with length $L=121$ and weight $w=11$. It is plotted in Fig. 4 versus different values of average photons per information nat. Other parameters used in the evaluations are as follows. The optical pulsewidth $\tau=10 \mathrm{~ns}$, the linewidth of the light source $\Delta \nu=2.5 \mathrm{THz}$, the coherence time $\tau_{c}=48.4 \mathrm{ps}$, the pulse-position multiplicity

$$
\begin{aligned}
P_{b}= & \frac{M}{2 M-1} P_{e} \\
\leq & \frac{2}{2 M-1} \sum_{h=h_{\min }}^{h_{\max }} \sum_{\boldsymbol{l} \in \mathcal{X}} P_{\boldsymbol{\kappa} \mid H}(\boldsymbol{l} \mid h) P_{H}(h) \sum_{m=0}^{M-2} \\
& \times \sum_{u=1}^{r_{\max }(m)} \operatorname{erfc}\left(\frac{\mu_{Y}\left(m, \boldsymbol{b}^{m,+} ; u\right)-\mu_{Z}\left(m+u, \boldsymbol{b}^{m,+} ; u\right)}{\sqrt{2\left[\sigma_{Y}^{2}\left(m, \boldsymbol{b}^{m,+}, \boldsymbol{l} ; u\right)+\sigma_{Z}^{2}\left(m+u, \boldsymbol{b}^{m,+}, \boldsymbol{l} ; u\right)\right]}}\right) \\
& \left.\quad+\sum_{u=r_{\max }(m)+1}^{M-1-m} \operatorname{erfc}\left(\frac{\mu_{I}\left(m, \boldsymbol{b}^{m,+}\right)-\mu_{I}\left(m+u, \boldsymbol{b}^{m,+}\right)}{\sqrt{2\left[\sigma_{I}^{2}\left(m, \boldsymbol{b}^{m,+}, \boldsymbol{l}\right)+\sigma_{I}^{2}\left(m+u, \boldsymbol{b}^{m,+}, \boldsymbol{l}\right)\right]}}\right)\right]
\end{aligned}
$$




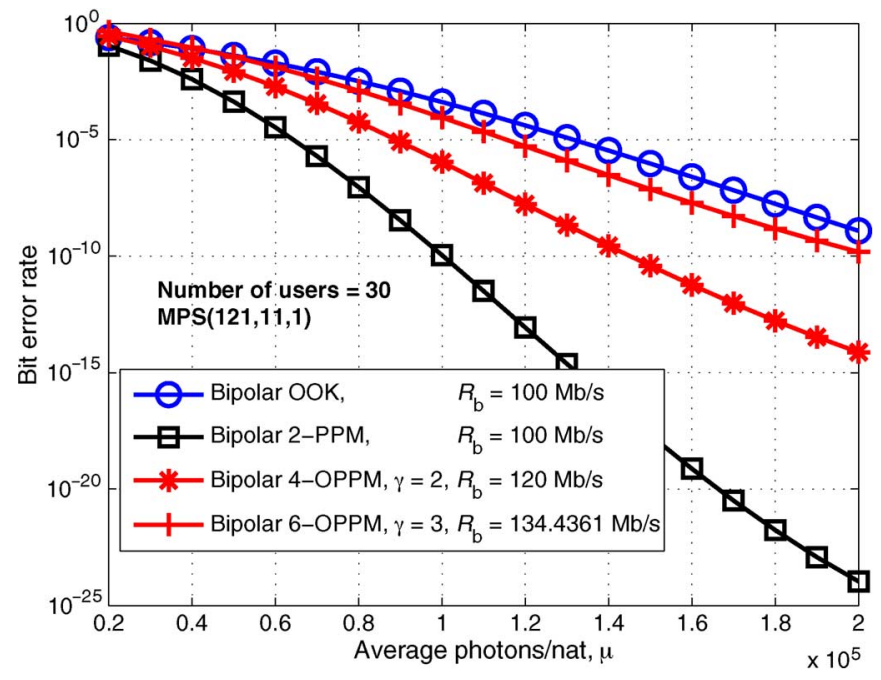

Fig. 4. Bit error rates of SAC-OCDMA systems versus average energy per nat for number of active users $N=30$ and optical pulsewidth $\tau=10 \mathrm{~ns}$.

$M \in\{4,6\}$ with corresponding index of overlap $\gamma \in\{2,3\}$, respectively, the receiver noise temperature $T^{\circ}=300 \mathrm{~K}$, the receiver load resistance $R_{L}=1 \mathrm{k} \Omega$, and the number of simultaneous users $N=30$ users. In addition, the BERs of both bipolar OOK and bipolar 2-PPM SAC-OCDMA systems, adopting same modified prime sequence code, are also plotted in the same figure for the sake of comparison. The superiority of our proposed technique under pulsewidth constraint is clear from the figure. Indeed, the transmission rate of the proposed system has increased by $20 \%$ and $34.44 \%$ (when using 4-OPPM and 6-OPPM techniques, respectively) above that of both OOK and 2-PPM techniques. Both bipolar OOKand PPM-CDMA systems have same transmission rate, which cannot be increased under the given pulsewidth constraint. In addition, the proposed system is more power efficient than traditional OOK-CDMA system. However, traditional PPM-CDMA system is more power efficient than the proposed system. In fact, the price paid to increase the transmission rate above that of PPM-CDMA is the increase in required energy for same BER. However, it is obvious that it is worth paying this price as the use of 2-PPM SAC-OCDMA system gives an over-improved BER, e.g., at an average photons per nat of $2 \times 10^{5}$, a BER of $10^{-24}$ is achieved by this system. On the other hand, 4-OPPM SAC-OCDMA system achieves a BER of $7.5 \times 10^{-15}$ with $20 \%$ increase in throughput for same average energy. It is also clear from the figure that increasing $M$, while keeping the ratio $\gamma / M$ fixed, would increase the transmission rate at the expense of reducing the BER even further. This is due to the increase of both the interference and the PIIN with the increase of the index of overlap.

Relaxing the constraint on the optical pulsewidth, would allow the data rate to increase in all systems as shown in Fig. 5 for $\tau=6.67 \mathrm{~ns}$. It is clear from the figure that both 2-OPPM (with $\gamma=2$ ) and OOK SAC-OCDMA systems coincide, with higher transmission rate of $33.33 \%$ in favor of the former. It is also clear from the figure that for fixed index of overlap and pulsewidth, the transmission rate increases with the decrease in $M$ at a price of worse BER. This is due to the loss of block

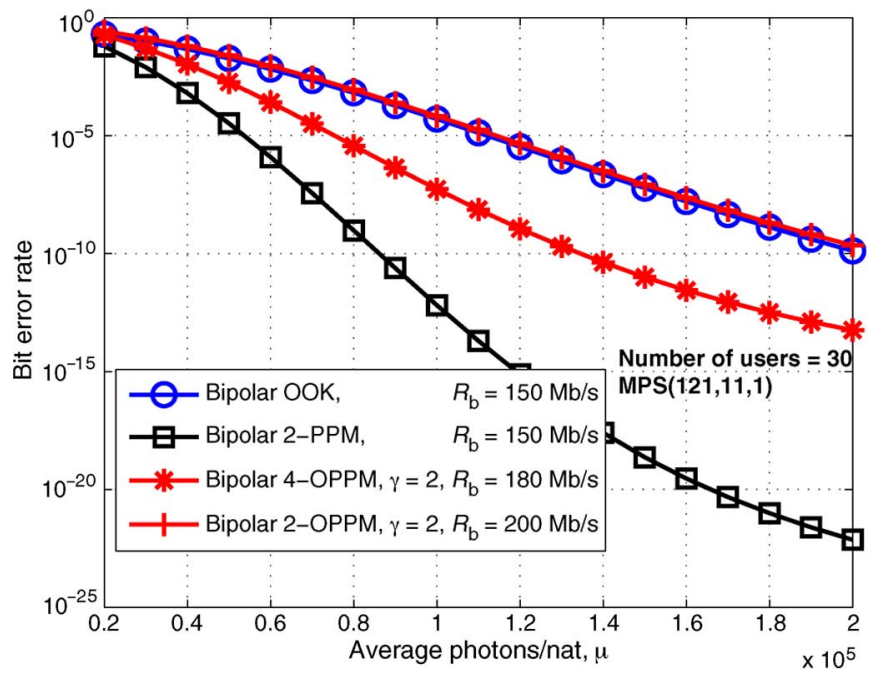

Fig. 5. Bit error rates of SAC-OCDMA systems versus average energy per nat for number of active users $N=30$ and optical pulsewidth $\tau=6.67 \mathrm{~ns}$.

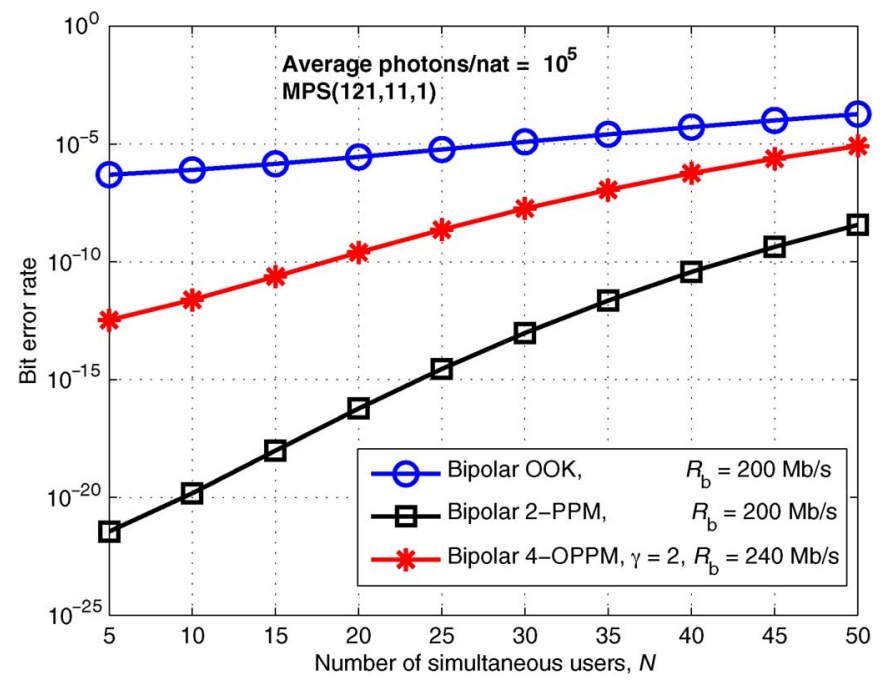

Fig. 6. Bit error rates of SAC-OCDMA systems versus number of active users for average energy per nat $\mu=10^{5}$ photons/nat and optical pulsewidth $\tau=$ $5 \mathrm{~ns}$.

coding gain with the decrease of $M$ and the decrease in the peak single-user power, cf. (38).

Finally in Fig. 6, we plot the BER versus the number of active users for same parameters as above but with $\tau=5 \mathrm{~ns}$ and average energy per nat $\mu=10^{5}$ photons/nat. It is obvious from the figure that the advantage of using the proposed method continues with the increase of the number of active users. However, the price paid also increases, as the BER of 4-OPPM SAC-OCDMA system becomes closer to that of OOK SAC-OCDMA system. This is because of the increase of interference in the case of OPPM more than that of the case of PPM due to the overlaps in the former.

\section{Conclusions}

A modulation technique has been proposed for SACOCDMA networks in order to increase the single-user transmission rate at a fixed pulsewidth. This technique allows an overlap between transmitted light pulses by adopting $M$-OPPM 

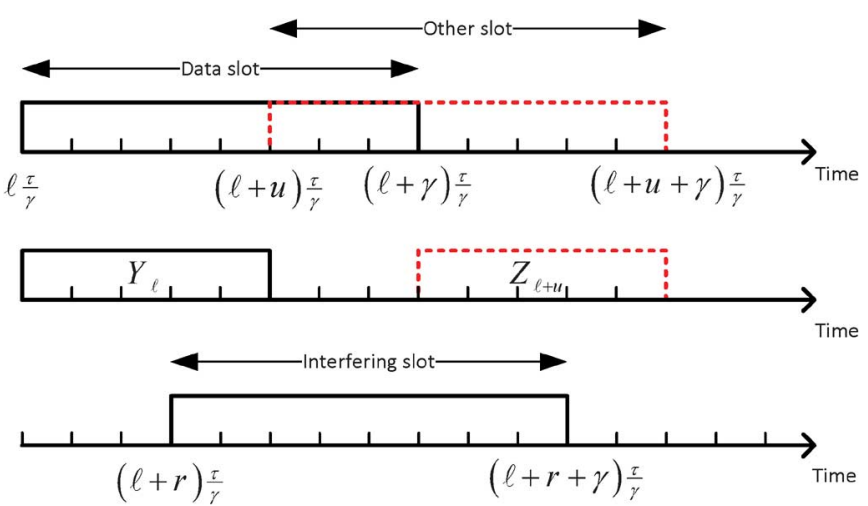

Fig. 7. Illustrations of different types of decision random variables within overlapped slots.

scheme to increase the transmission rate in a reliable way. Each pulse can take two different levels, represented by two orthogonal code sequences. The bit error rate (BER) of the proposed system has been derived and compared numerically to that of corresponding systems adopting $M$-PPM and OOK schemes. It turned out that under pulsewidth constraint, the proposed $M$-OPPM SAC-OCDMA system achieves higher transmission rate than that achieved by both $M$-PPM and OOK SAC-OCDMA systems, while keeping the BER below a prescribed threshold.

\section{APPENDIX A \\ EXTENSION TO THE CASE OF $u<\gamma$}

During the decision process, random variable $I_{m}, m \in \mathcal{M}$, is compared to $I_{m+u}$ for $u \in\{1,2, \ldots, M-1-m\}$, cf. (35). The derivation given in Section III-A has been done assuming that there is no overlap between slots $m$ and $m+u$. This is valid as long as $u \in\left\{r_{\max }(m)+1, \ldots, M-1-m\right\}$. However, in case of $u \in\left\{1,2, \ldots, r_{\max }(m)\right\}$, this comparison involves an overlapping part as shown in Fig. 7 with $m=\ell$. To get the statistics of the non-overlapped slots, we consider two cases, data slot and other slot, as follows.

\section{A. Case of Data Slot}

Because of the overlap between data slot $m=\ell$ and other slot $m+u=\ell+u$, we consider the following two random processes for data slot $m=\ell$ of Fig. 7:

$$
\begin{aligned}
\zeta_{n, \ell}^{i}(t) \stackrel{\text { def }}{=} & \xi_{n, \ell}^{i}(t) \cdot g\left[\frac{t-\ell \frac{\tau}{\gamma}}{u \frac{\tau}{\gamma}}\right] \\
= & \sum_{r=r_{\min }(\ell)}^{r_{\text {max }}(\ell)}\left[\alpha_{n, \ell+r} c_{n}(i)+\beta_{n, \ell+r} c_{n}^{\prime}(i)\right] \\
& \times g\left[\frac{t-(\ell+r \vee 0) \frac{\tau}{\gamma}}{a_{r u} \tau}\right]
\end{aligned}
$$

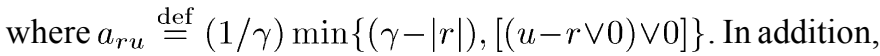
we define the following two random variables:

$$
\begin{aligned}
& Y_{U, \ell} \stackrel{\text { def }}{=} \frac{\mathcal{R} P_{0}}{L} \sum_{i=1}^{L}\left[\sum_{n=1}^{N} c_{1}(i) \frac{1}{\tau} \int_{\ell \tau / \gamma}^{(\ell / \gamma+1) \tau} \zeta_{n, \ell}^{i}(t) d t\right. \\
& +2 \sum_{n=1}^{N-1} \sum_{m=n+1}^{N} c_{1}(i) \frac{1}{\tau} \\
& (\ell / \gamma+1) \tau \\
& \int_{\ell \tau / \gamma} \zeta_{n, \ell}^{i}(t) \zeta_{m, \ell}^{i}(t) \\
& \left.\times \cos \left(\phi_{n i}(t)-\phi_{m i}(t)\right) d t\right] \\
& Y_{L, \ell} \stackrel{\text { def }}{=} \frac{\mathcal{R} P_{0}}{L} \sum_{i=1}^{L}\left[\sum_{n=1}^{N} c_{1}^{\prime}(i) \frac{1}{\tau} \int_{\ell \tau / \gamma}^{(\ell / \gamma+1) \tau} \zeta_{n, \ell}^{i}(t) d t\right. \\
& +2 \sum_{n=1}^{N-1} \sum_{m=n+1}^{N} c_{1}^{\prime}(i) \frac{1}{\tau} \\
& (\ell / \gamma+1) \tau \\
& \int_{\ell \tau / \gamma} \zeta_{n, \ell}^{i}(t) \zeta_{m, \ell}^{i}(t) \\
& \left.\times \cos \left(\phi_{n i}(t)-\phi_{m i}(t)\right) d t\right] .
\end{aligned}
$$

Following a similar analysis to what have been done in Section III-A, we get the mean and variance of random variable $Y_{\ell} \stackrel{\text { def }}{=} Y_{U, \ell}-Y_{L, \ell}$ as follows:

$$
\begin{aligned}
& \mu_{Y}(\ell, \boldsymbol{b} ; u) \\
& =\frac{\mathcal{R} P_{0}}{L} w \sum_{r=r_{\min }(\ell)}^{r_{\text {max }}(\ell)} a_{r u} b_{\ell+r} \\
& \sigma_{Y}^{2}(\ell, \boldsymbol{b}, \boldsymbol{l} ; u) \\
& =\sigma_{T}^{2}+4 B_{e} \tau_{c}\left(\frac{\mathcal{R} P_{0}}{L}\right)^{2}\left[\sum_{r=r_{\text {min }}(\ell)} \sum_{s=r_{\text {min }}(\ell)}^{r_{\text {max }}(\ell)} b_{r s u}\right. \\
& \times\left(\lambda\left|b_{\ell+r}\right|+\frac{\lambda w}{L} \cdot l_{\ell+r}\right) l_{\ell+s} \\
& \left.-\sum_{r=r_{\min }(\ell)}^{r_{\text {max }}(\ell)} a_{r u} \frac{\lambda w}{L} \cdot l_{\ell+r}\right] \\
& +2 e B_{e} \frac{\mathcal{R} P_{0}}{L}\left[\sum_{r=r_{\text {min }}(\ell)}^{r_{\text {max }}(\ell)} a_{r u}\left(w\left|b_{\ell+r}\right|+2 \lambda l_{\ell+r}\right)\right], \quad(\mathrm{A} .3)
\end{aligned}
$$

respectively, where $b_{\text {rsu }} \stackrel{\text { def }}{=}(1 / \gamma) \min \{(\gamma-\max \{|r|,|s|, \mid r-$ $s \mid \wedge \gamma\}),(u-u \wedge \max \{r, s, 0\})\}$. 


\section{B. Case of Other Slot}

Because of the overlap between other slot $m=\ell$ and data slot $m-u=\ell-u$, we consider the following two random processes for other slot $m=\ell$ (this is similar to other slot of Fig. 7 but shifted to the left by $u$ ):

$$
\begin{aligned}
\varsigma_{n, \ell}^{i}(t) \stackrel{\text { def }}{=} & \xi_{n, \ell}^{i}(t) \cdot g\left[\frac{t-(\ell+\gamma-u) \frac{\tau}{\gamma}}{u \frac{\tau}{\gamma}}\right] \\
= & \sum_{r=r_{\min }(\ell)}^{r_{\max }(\ell)}\left[\alpha_{n, \ell+r} c_{n}(i)+\beta_{n, \ell+r} c_{n}^{\prime}(i)\right] \\
& \times g\left[\frac{t-(\ell+r \vee(\gamma-u) \vee 0) \frac{\tau}{\gamma}}{\min \{(\gamma-|r|),[(u+r \wedge 0) \vee 0]\} \frac{\tau}{\gamma}}\right] \\
= & \sum_{r=r_{\min }(\ell)}^{r_{\max }(\ell)}\left[\alpha_{n, \ell+r} c_{n}(i)+\beta_{n, \ell+r} c_{n}^{\prime}(i)\right] \\
& \times g\left[\frac{t-(\ell+r \vee(\gamma-u) \vee 0) \frac{\tau}{\gamma}}{a_{(-r) u} \tau}\right]
\end{aligned}
$$

In addition, we define the following two random variables:

$$
\begin{aligned}
& Z_{U, \ell} \stackrel{\text { def }}{=} \frac{\mathcal{R} P_{0}}{L} \sum_{i=1}^{L}\left[\sum_{n=1}^{N} c_{1}(i) \frac{1}{\tau} \int_{\ell \tau / \gamma}^{(\ell / \gamma+1) \tau} \varsigma_{n, \ell}^{i}(t) d t\right. \\
& +2 \sum_{n=1}^{N-1} \sum_{m=n+1}^{N} c_{1}(i) \frac{1}{\tau} \\
& (\ell / \gamma+1) \tau \\
& \int_{\ell \tau / \gamma} \varsigma_{n, \ell}^{i}(t) \varsigma_{m, \ell}^{i}(t) \\
& \left.\times \cos \left(\phi_{n i}(t)-\phi_{m i}(t)\right) d t\right] \\
& Z_{L, \ell} \stackrel{\text { def }}{=} \frac{\mathcal{R} P_{0}}{L} \sum_{i=1}^{L}\left[\sum_{n=1}^{N} c_{1}^{\prime}(i) \frac{1}{\tau} \int_{\ell \tau / \gamma}^{(\ell / \gamma+1) \tau} \varsigma_{n, \ell}^{i}(t) d t\right. \\
& +2 \sum_{n=1}^{N-1} \sum_{m=n+1}^{N} c_{1}^{\prime}(i) \frac{1}{\tau} \\
& (\ell / \gamma+1) \tau \\
& \int_{\ell \tau / \gamma} \varsigma_{n, \ell}^{i}(t) \varsigma_{m, \ell}^{i}(t) \\
& \left.\times \cos \left(\phi_{n i}(t)-\phi_{m i}(t)\right) d t\right] .
\end{aligned}
$$

Following a similar analysis to what have been done in Section III-A, we get the mean and variance of the random variable $Z_{\ell} \stackrel{\text { def }}{=} Z_{U, \ell}-Z_{L, \ell}$ as follows:

$$
\begin{aligned}
& \mu_{Z}(\ell, \boldsymbol{b} ; u) \\
& =\frac{\mathcal{R} P_{0}}{L} w \sum_{r=r_{\min }(\ell)}^{r_{\text {max }}(\ell)} a_{(-r) u} b_{\ell+r} \\
& \sigma_{Z}^{2}(\ell, \boldsymbol{b}, \boldsymbol{l} ; u) \\
& =\sigma_{T}^{2}+4 B_{e} \tau_{c}\left(\frac{\mathcal{R} P_{0}}{L}\right)^{2} \\
& \times\left[\sum_{r=r_{\min }(\ell)}^{r_{\max }(\ell)} \sum_{s=r_{\min }(\ell)}^{r_{\max }(\ell)} b_{(-r)(-s) u}\right. \\
& \times\left(\lambda\left|b_{\ell+r}\right|+\frac{\lambda w}{L} \cdot l_{\ell+r}\right) l_{\ell+s} \\
& \left.-\sum_{r=r_{\min }(\ell)}^{r_{\max }(\ell)} a_{(-r) u} \frac{\lambda w}{L} \cdot l_{\ell+r}\right] \\
& +2 e B_{e} \frac{\mathcal{R} P_{0}}{L}\left[\sum_{r=r_{\min }(\ell)}^{r_{\max }(\ell)} a_{(-r) u}\left(w\left|b_{\ell+r}\right|+2 \lambda l_{\ell+r}\right)\right] \text {. }
\end{aligned}
$$

\section{REFERENCES}

[1] T. A. Bhuiyan, M. Z. Hassan, S. M. S. Tanzil, S. Hayder, and S. P. Majumder, "Performance improvement of IM-DD free space optical CDMA (attenuated by strong atmospheric turbulence) with maximal ratio combining," in Proc. IEEE Int. Conf. Comput. Intel. Commun. Net. (CICN 2010), Bhopal, India, Nov. 26-28, 2010, pp. 513-518.

[2] S.-P. Tseng and J. Wu, "The SAC OCDMA PON with super perfect difference codes," in Proc. IEEE Int. Conf. Commun. (ICC 2011), Kyoto, Japan, Jun. 5-9, 2011, pp. 1-4.

[3] C. Y. Chang, G. C. Yang, C. Y. Chang, and W. C. Kwong, "Study of a diversity O-CDMA scheme for optical wireless," J. Lightw. Technol., vol. 30, pp. 1549-1558, May 15, 2012.

[4] H. Beyranvand and J. A. Salehi, "Efficient optical resource allocation and QoS differentiation in optical burst switching networks utilizing hybrid WDM/OCDM," J. Lightw. Technol., vol. 30, no. 15, pp. 2427-2441, Aug. 1, 2012.

[5] Y.-K. Choi, M. Hanawa, X. Wang, C.-S. Park, and C.-S. , "Upstream transmission of WDM/OCDM-PON in a loop-back configuration with remotely supplied short optical pulses," IEEE/OSA J. Opt. Commun. Net., vol. 5, no. 3, pp. 183-189, Mar. 1, 2013.

[6] J. Penon, Z. A. El-Sahn, L. A. Rusch, and S. LaRochelle, "Spectral-amplitude-coded OCDMA optimized for a realistic FBG frequency response," J. Lightw. Technol., vol. 25, no. 5, pp. 1256-1263, May 2007.

[7] D. Zaccarin and M. Kavehrad, "An optical CDMA system based on spectral encoding of LED," IEEE Photon. Technol. Lett., vol. 4, pp. 479-482, Apr. 1993.

[8] D. Zaccarin and M. Kavehrad, "Performance evaluation of optical CDMA systems using non-coherent detection and bipolar codes," $J$. Lightw. Technol., vol. 12, no. 1, pp. 96-105, Jan. 1994.

[9] E. D. J. Smith, R. J. Blaikie, and D. P. Taylor, "Performance enhancement of spectral-amplitude-coding optical CDMA using pulse-position modulation," IEEE Trans. Commun., vol. 46, no. 9, pp. 1176-1185, Sep. 1998.

[10] X. Zhou, H. M. H. Shalaby, and C. Lu, "Design and performance analysis of a new code for spectral-amplitude-coding optical CDMA systems," in Proc. IEEE Sixth Int. Symp. Spread Spectrum Tech. Applications (ISSSTA 2000), Sep. 6-8, 2000, pp. 174-178. 
[11] Z. Wei, H. M. H. Shalaby, and H. Ghafouri-Shiraz, "Modified quadratic congruence codes for fiber Bragg-grating-based spectral-amplitude-coding optical CDMA systems," J. Lightw. Technol., vol. 19, pp. 1274-1281, Sept. 2001.

[12] Z. Wei and H. Ghafouri-Shiraz, "Proposal of a novel code for spectral amplitude-coding optical CDMA systems," IEEE Photon. Technol. Lett., vol. 14, no. 3, pp. 414-416, Mar. 2002.

[13] Z. Wei and H. Ghafouri-Shiraz, "Codes for spectral-amplitude-coding optical CDMA systemss," J. Lightw. Technol., vol. 20, no. 8, pp. 1284-1291, Aug. 2002.

[14] H. M. H. Shalaby, "Efficient use of PPM in spectral-amplitude-coding optical CDMA systems," J. Lightw. Technol., vol. 30, no. 22, pp. 3512-3519, Nov. 15, 2012.

[15] W. C. Kwong, P. A. Perrier, and P. R. Prucnal, "Performance comparison of asynchronous and synchronous code-division multiple-access," IEEE Trans. Commun., vol. COM-39, pp. 1625-1634, Nov. 1991.

[16] M. Tavan, E. Agrell, and J. Karout, "Bandlimited intensity modulation," IEEE Trans. Commun., vol. 60, no. 11, pp. 3429-3439, Nov. 2012.

[17] T. Lueftner, C. Kroepl, M. Huemer, J. Hausner, R. Hagelauer, and R. Weigel, "Edge-position modulation for high-speed wireless infrared communications," Optoelectronics, IEE Proc., vol. 150, no. 5, pp. 427-437, Oct. 17, 2003.

[18] R. E. Freund, C. A. Bunge, N. N. Ledentsov, D. Molin, and C. Caspar, "High-speed transmission in multimode fibers," J. Lightw. Technol., vol. 28 , no. 4, pp. 569-586, Feb. 2010.

[19] J. M. Kahn and J. R. Barry, "Wireless infrared communications," Proc. IEEE, vol. 85, no. 2, pp. 265-298, Feb. 1997.

[20] G. M. Lee and G. W. Schroeder, "Optical PPM with multiple positions per pulse-width," IEEE Trans. Commun., vol. COM-25, no. 3 , pp. 360-364, Mar. 1977

[21] I. Bar-David and G. Kaplan, "Information rates of photon-limited overlapping pulse position modulation channels," IEEE Trans. In. Theory, vol. IT-30, pp. 455-464, May 1984.

[22] C. N. Georghiades, "Some implications of TCM for optical direct detection channels," IEEE Trans. Commun., vol. COM-37, pp. 481-487, May 1989.

[23] C. N. Georghiades, "Modulation and coding for throughput-efficient optical systems," IEEE Inf. Theory, vol. IT-40, pp. 1313-1326, Sept. 1994.

[24] H. M. H. Shalaby, "Maximum achievable throughputs for uncoded OPPM and MPPM in optical direct-detection channels," J. Lightw. Technol., vol. LT-13, pp. 2121-2128, Nov. 1995.

[25] J. Magné, D.-P. Wei, S. Ayotte, L. A. Rusch, and S. LaRochelle, "Experimental demonstration of frequency-encoded optical CDMA using superimposed fiber Bragg gratings," in Proc. Conf. Bragg Gratings, Poling \& Photosensitivity in Glass Waveguides (BGPP 2003), Monterey, CA, Sep. 1, 2003, pp. 294-296.
[26] S. Ayotte, M. J. Magné, L. A. Rusch, and S. LaRochelle, "Experimental verification and capacity prediction of FE-OCDMA using superimposed FBG," J. Lightw. Technol., vol. 23, no. 2, pp. 724-731, Feb. 2005

[27] M. M. Rad and J. A. Salehi, "Phase-induced intensity noise in digital incoherent all-optical tapped-delay line systems," J. Lightw. Technol., vol. 24, no. 8, pp. 3059-3072, Aug. 2006.

[28] M. Noshad and K. Jamshidi, "Bounds for the BER of codes with fixed cross correlation in SAC-OCDMA systems," J. Lightw. Technol., vol. 29, no. 13, pp. 1944-1950, July 2011.

[29] H. M. H. Shalaby, "Closed form expression for the bit-error rate of spectral-amplitude-coding optical CDMA systems," IEEE Photon. Technol. Lett., vol. 24, pp. 1285-1287, Aug. 1, 2012.

Hossam M. H. Shalaby (S'83-M'91-SM'99) was born in Giza, Egypt, in 1961. He received the B.S. and M.S. degrees from Alexandria University, Alexandria, Egypt, in 1983 and 1986, respectively, and the Ph.D. degree from the University of Maryland at College Park in 1991, all in electrical engineering.

In 1991, he joined the Electrical Engineering Department, Alexandria University, and was promoted to Professor in 2001. Currently he is on leave from Alexandria University, where he is the chair of the Department of Electronics and Communications Engineering, School of Electronics, Communications, and Computer Engineering, Egypt-Japan University of Science and Technology (E-JUST), New Borg EL-Arab City, Alexandria, Egypt. From December 2000 to 2004, he was an Adjunct Professor with the Faculty of Sciences and Engineering, Department of Electrical and Information Engineering, Laval University, Quebec, QC, Canada. From September 1996 to February 2001, he was on leave from the Alexandria University. From September 1996 to January 1998, he was with the Electrical and Computer Engineering Department, International Islamic University Malaysia, and from February 1998 to February 2001, he was with the School of Electrical and Electronic Engineering, Nanyang Technological University, Singapore. His research interests include optical communications, optical CDMA, optical burst-switching, OFDM technology, and information theory.

Prof. Shalaby has served as a student branch counselor at Alexandria University, IEEE Alexandria and North Delta Subsection, from 2002 to 2006, and served as a chairman of the student activities committee of IEEE Alexandria Subsection from 1995 to 1996 . He received an SRC fellowship from 1987 to 1991 from Systems Research Center, Maryland; State Excellence Award in Engineering Sciences in 2007 from Academy of Scientific Research and Technology, Egypt; Shoman Prize for Young Arab Researchers in 2002 from Abdul Hameed Shoman Foundation, Amman, Jordan; State Incentive Award in Engineering Sciences in 1995 and 2001 from Academy of Scientific Research and Technology, Egypt; University Excellence Award in 2009 from Alexandria University; and University Incentive Award in 1996 from Alexandria University. $\mathrm{He}$ is a member of the IEEE Photonics Society and The Optical Society (OSA). 